\title{
Landau levels and oscillator strength in a biased bilayer of graphene
}

\author{
J. Milton Pereira, Jr., ${ }^{1,2}$ F. M. Peeters, ${ }^{1}$ and P. Vasilopoulos ${ }^{3}$ \\ ${ }^{1}$ Department of Physics, Universiteit Antwerpen Groenenborgerlaan 171, B-2020 Antwerpen, Belgium \\ ${ }^{2}$ Departamento de Física, Universidade Federal do Ceará, Fortaleza, Ceará 60455-760, Brazil \\ ${ }^{3}$ Department of Physics, Concordia University, Montreal, Quebec, Canada H3G 1M8
}

(Received 1 June 2007; published 14 September 2007)

\begin{abstract}
We obtain analytical expressions for the eigenstates and the Landau level spectrum of biased graphene bilayers in a magnetic field. The calculations are performed in the context of a four-band continuum model and generalize previous approximate results. Solutions are presented for the spectrum as a function of interlayer coupling, the potential difference between the layers, and the magnetic field. The explicit expressions allow us to calculate the oscillator strength and the selection rules for electric dipole transitions between the Landau states. Some transitions are significantly shifted in energy relative to those in an unbiased bilayer and exhibit a very different magnetic field dependence.
\end{abstract}

DOI: 10.1103/PhysRevB.76.115419

PACS number(s): 71.10.Pm, 73.21.-b, 81.05.Uw

\section{INTRODUCTION}

The recent realization of stable single layer and bilayer carbon crystals (graphene) has aroused considerable interest in the study of their electronic properties. ${ }^{1-5}$ These materials have been shown to display interesting new phenomena that may allow the development of carbon-based nanoelectronic devices. The behavior of charge carriers in wide single-layer graphene sheets has been described as that of ultrarelativistic massless particles, with a "light speed" equal to the Fermi velocity of the crystal and a gapless linear dispersion close to the $K$ and $K^{\prime}$ points. One consequence of that is that singlelayer graphene displays an unusual quantum Hall effect, in which the quantum Hall plateaus are found in half-integer multiples of $4 e^{2} / h$. Moreover, the massless character of the charge carriers in single-layer graphene gives a $\sqrt{B}$ dependence to the Landau levels (LLs), which has been recently confirmed by infrared transmission and cyclotron resonance experiments in thin graphite samples ${ }^{6}$ and on single layers of graphene. ${ }^{7}$ On the other hand, for an unbiased graphene bilayer, the spectrum at the vicinity of the $K$ points displays four parabolic bands but is still gapless. ${ }^{8,9}$ The absence of a gap, together with the chiral nature of the electronic states, in both single-layer and bilayer graphene is at the root of phenomena, such as the Klein tunneling, ${ }^{10-12}$ which has important consequences for the design of graphene-based devices. ${ }^{13}$

Recent theoretical and experimental results have shown that a gap can be induced in bilayer graphene by changing the density of charge carriers in the layers through the application of an external field or by chemical doping, which creates a potential difference between the layers. ${ }^{14,15}$ The presence of such a bias transforms the graphene bilayer into a semiconductor with a tunable gap. This implies that such biased bilayers can be used to confine charge carriers through the use of, e.g., nanostructured gates or spatially varying doping. ${ }^{16}$ Tight-binding calculations have shown that the existence of such a gap can have a significant effect on the LL spectrum of biased graphene bilayers. ${ }^{17,18}$

In this work, we determine the spectrum of a biased graphene bilayer in the presence of an external magnetic field in the continuum description and obtain, by solving the four-band Hamiltonian, the exact eigenstates as well as the oscillator strengths and the selection rules for the electric dipole transitions between LL. Previous numerical results for the LL in biased ${ }^{18}$ and unbiased ${ }^{19}$ bilayer graphene, were obtained within a model based on a reduced two-band Hamiltonian, which is accurate for energies much lower than the interlayer coupling parameter. Dipole transitions were not studied in Ref. 18 but were in Ref. 20 for unbiased layers. In addition, these previous studies used a description in which the Hamiltonian can be described approximately in terms of ladder operators on the basis of Landau functions, which is only possible in the Landau gauge. Here, we generalize these results to much higher energy by using the more accurate four-band model, and obtain exact expressions in both the Landau gauge and the symmetric gauge. The calculations allow us to obtain a significant correction to the spectrum obtained by previous approximate methods. The model is presented in Sec. II for zero magnetic field $B$. A finite field $B$ is considered in Sec. III, in the Landau and symmetric gauges, and the results are given in Sec. IV. The oscillator strength is evaluated in Sec. V and a summary follows in Sec. VI.

\section{ZERO MAGNETIC FIELD}

The crystal structure of an undoped bilayer of graphene is that of two honeycomb sheets of covalent-bond carbon atoms coupled by weak Van der Waals forces. To each carbon atom corresponds a valence electron, and the structure can be described in terms of four sublattices, labeled A, B (upper layer) and $\mathrm{A}^{\prime}, \mathrm{B}^{\prime}$ (lower layer). In this work, we focus on the Bernal stacking, in which half the atoms of the upper layer (i.e., the sites of the A sublattice) are on top of half the sites of the lower layer (the sites of the B' sublattice). This type of stacking is found in graphite and has been reported as well for samples of bilayers of graphene. Alternatively, one can consider the hexagonal stacking, in which all sites of the top layer are on top of all sites of the lower layer. That configuration introduces a different coupling scheme between the layers, and can lead to qualitatively distinct results. However, 
the band structure data from the epitaxial bilayer samples of Ref. 14, as well as the results from the samples produced by micromechanical cleavage of graphite in Ref. 18 are consistent with the Bernal stacking. The coupling between the layers is described by an interaction term between the $A$ and $B^{\prime}$ sublattice sites. Considering only nearest-neighbor hopping, the Hamiltonian of the system in the vicinity of the $K$ point is given, in the continuum approximation, by ${ }^{20}$

$$
\mathcal{H}=\mathcal{H}_{0}+(\Delta U / 2) \tau_{z}
$$

with

$$
\mathcal{H}_{0}=\left(\begin{array}{cccc}
U_{0} & \pi & t & 0 \\
\pi^{\dagger} & U_{0} & 0 & 0 \\
t & 0 & U_{0} & \pi^{\dagger} \\
0 & 0 & \pi & U_{0}
\end{array}\right)
$$

the operator $\tau_{z}$ assigns a positive (negative) sign to the upper (lower) layer labels and is defined as

$$
\tau_{z}=\left(\begin{array}{cc}
\mathbf{I} & 0 \\
0 & -\mathbf{I}
\end{array}\right),
$$

with I denoting the $2 \times 2$ identity matrix. We assume a constant interlayer coupling term $t \approx 400 \mathrm{meV} ; \pi=v_{F}\left(p_{x}+i p_{y}\right)$, $\hat{\mathbf{p}}=\left(p_{x}, p_{y}\right)$ is the two-dimensional (2D) momentum operator, $v_{F} \approx 1 \times 10^{6} \mathrm{~m} / \mathrm{s}, U_{0}=\left(U_{1}+U_{2}\right) / 2, \Delta U=U_{1}-U_{2}$, and $U_{1}, U_{2}$ are the potentials at the two layers, which reflect the influence of doping on one of them and/or the interaction with an external electric field, i.e., the gating. In this work, we treat the potentials at each layer and the gap as adjustable parameters, which may be obtained experimentally, e.g., from angle-resolved photoemission spectroscopy measurements. ${ }^{14}$ The eigenstates of Eq. (1) are fourcomponent spinors $\Psi=\left[\psi_{A}, \psi_{B}, \psi_{B^{\prime}}, \psi_{A^{\prime}}\right]^{T}$, where $\psi_{A, B}$ $\left(\psi_{A^{\prime}, B^{\prime}}\right)$ are the envelope functions associated with the probability amplitudes at the respective sublattice sites of the upper (lower) graphene sheet. The superscript $T$ denotes the transpose of the $[\ldots]$ vector.

If the magnetic field is absent and the potentials $U_{1}$ and $U_{2}$ are constant, the single-particle spectrum consists of four bands with eigenvalues ${ }^{16}$

$$
\begin{aligned}
& E_{ \pm}^{+}(k)=U_{0}+(1 / 2)\left[(t \pm \Gamma)^{2}+\Omega\right]^{1 / 2}, \\
& E_{ \pm}^{-}(k)=U_{0}-(1 / 2)\left[(t \pm \Gamma)^{2}+\Omega\right]^{1 / 2},
\end{aligned}
$$

where $\Gamma=\left[t^{2}+4 s_{F}^{2}+4\left(s_{F}^{2} / t^{2}\right) \Delta U^{2}\right]^{1 / 2}, \quad s_{F}=\hbar v_{F} k$, and $\Omega=[1$ $\left.-4 s_{F}^{2} / t^{2}\right] \Delta U^{2}$. Note that for $k=0$, the spectrum shows a gap at $k=0$ of size $E_{-}^{+}(0)-E_{-}^{-}(0)=|\Delta U|$ and the system becomes a narrow-gap semiconductor. Figure 1 shows the low-energy electronic spectrum of graphene bilayers for $U_{0}=0$ and three different values of the gap: $\Delta U=0$ (black solid line), $\Delta U$ $=25 \mathrm{meV}$ (red dashed line), $\Delta U=100 \mathrm{meV}$ (blue dotted), and $\Delta U=200 \mathrm{meV}$ (green dot-dashed line). For large $k$ values, the linear $E-k$ behavior is recovered. The hole spectrum in this case is obtained by taking $E(k) \rightarrow-E(k)$. In the absence of bias, the spectrum shows approximately parabolic bands, which become increasingly deformed as $\Delta U$ increases, with the appearance of energy minima at nonzero

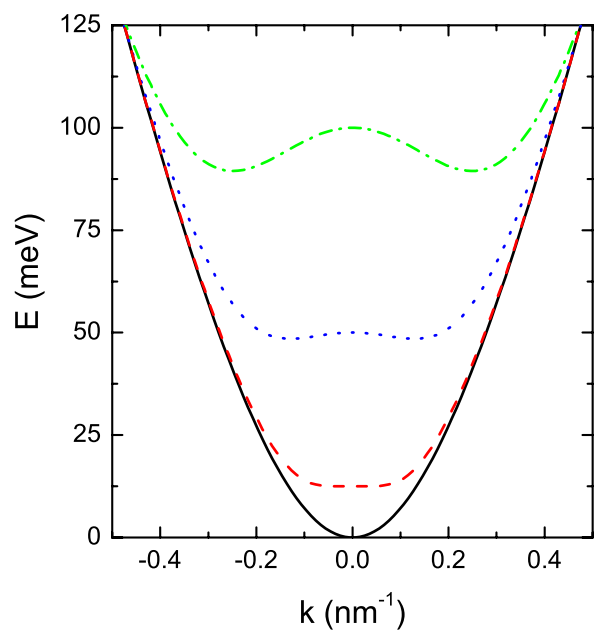

FIG. 1. (Color online) Low-energy electronic dispersion of graphene bilayers for $B=0$ for different values of the gap, namely, $\Delta U=0$ (black solid line), $\Delta U=25 \mathrm{meV}$ (red dashed line), $\Delta U$ $=100 \mathrm{meV}$ (blue dotted), and $\Delta U=200 \mathrm{meV}$ (green dot-dashed line). In all cases, $U_{0}=0$.

values of $k$, and can be approximated by a fourth-order polynomial function. ${ }^{8,21}$ As we show below, this nonparabolic dispersion has a significant influence on the LL results.

\section{FINITE MAGNETIC FIELD}

We consider an undoped bilayer of graphene in the presence of an external perpendicular magnetic field $B$. It is instructive to consider separately the two gauges for the vector potential A given below. The momentum operator $\mathbf{p}$, appearing in the term $\mathcal{H}_{0}$ in Eq. (1), is shifted by $-e \mathbf{A}$.

\section{A. Landau gauge}

In this gauge, we have $\mathbf{A}=(0, B x, 0)$ with $\left[\mathcal{H}_{0}, p_{y}\right]=0$. Consequently, we can write the solutions for the spinor components in the form $\psi_{A}(x, y)=\phi_{A}(x) e^{i k_{y} y}, \quad \psi_{B}(x, y)$ $=i \phi_{B}(x) e^{i k_{y} y}, \quad \psi_{A^{\prime}}(x, y)=i \phi_{A^{\prime}}(x) e^{i k_{y} y}, \quad$ and $\quad \psi_{B^{\prime}}(x, y)$ $=\phi_{B^{\prime}}(x) e^{i k_{y} y}$. The $x$ dependence of the spinor components is then described by

$$
\begin{gathered}
\hbar v_{F}\left(\frac{d}{d x}+k_{y}+\beta x\right) \phi_{A}=\left(E-U_{1}\right) \phi_{B}, \\
\hbar v_{F}\left(\frac{d}{d x}-k_{y}-\beta x\right) \phi_{B}=-\left(E-U_{1}\right) \phi_{A}+t \phi_{B^{\prime}}, \\
\hbar v_{F}\left(\frac{d}{d x}+k_{y}+\beta x\right) \phi_{A^{\prime}}=-\left(E-U_{2}\right) \phi_{B^{\prime}}+t \phi_{A}, \\
\hbar v_{F}\left(\frac{d}{d x}-k_{y}-\beta x\right) \phi_{B^{\prime}}=\left(E-U_{2}\right) \phi_{A^{\prime}},
\end{gathered}
$$

where $\beta=e B / \hbar$. For a wide, uniform system, these equations can be decoupled to obtain, for $\phi_{A}$, 


$$
\left(\frac{d^{2}}{d \xi^{2}}+\alpha^{2}+\delta^{2}-\xi^{2}\right)^{2} \phi_{A}=\left\{\left(\alpha^{2}-\delta^{2}\right) t^{\prime 2}+[1-2 \delta \alpha]^{2}\right\} \phi_{A},
$$

with $\xi=\ell_{B} k_{y}+x / \ell_{B}$; the characteristic length scale of the system is the magnetic length $\ell_{B}=[\hbar / e B]^{1 / 2}, \alpha=\epsilon-u_{0}$ is the energy shifted by the average potential between the layers $u_{0}=\left(u_{1}+u_{2}\right) / 2$, and $\delta=\Delta u / 2$, where $\Delta u=u_{1}-u_{2}$. The energy, the potentials, and the interlayer coupling strength are written in dimensionless units as $\epsilon=E \ell_{B} / \hbar v_{F}, u_{1,2}=U_{1,2} \ell_{B} / \hbar v_{F}$, and $t^{\prime}=t \ell_{B} / \hbar v_{F}$, respectively. Next, we consider the equation

$$
\left(-\partial^{2} / \partial \xi^{2}+\xi^{2}\right) \phi_{A}=\gamma_{ \pm}(\alpha) \phi_{A},
$$

where the potential-dependent eigenvalues are

$$
\gamma_{ \pm}(\alpha)=\alpha^{2}+\delta^{2} \pm\left[\left(\alpha^{2}-\delta^{2}\right) t^{\prime 2}+(1-2 \delta \alpha)^{2}\right]^{1 / 2} .
$$

With the substitution $\phi_{A}=f_{A} \exp \left(-\xi^{2} / 2\right)$, and after some straightforward algebra, the LL spectrum is given by the solutions $\alpha_{n}$ of the fourth-order algebraic equation

$$
\left[(\alpha+\delta)^{2}-2(n+1)\right]\left[(\alpha-\delta)^{2}-2 n\right]-\left(\alpha^{2}-\delta^{2}\right) t^{\prime 2}=0,
$$

where $n$ is an integer. The solutions of Eq. (11) can be obtained in terms of Hermite polynomials.

Substituting $\phi_{A}$ in Eqs. (6)-(9), we obtain

$$
\begin{gathered}
\phi_{A}=d_{n} c_{n} H_{n}(\xi) e^{-\xi^{2} / 2}, \\
\phi_{B}=d_{n} \frac{\sqrt{2 n}}{\alpha_{n}-\delta} c_{n-1} H_{n-1}(\xi) e^{-\xi^{2} / 2}, \\
\phi_{A^{\prime}}=-f_{n} d_{n} \frac{\sqrt{2(n+1)}}{\alpha_{n}+\delta} c_{n+1} H_{n+1}(\xi) e^{-\xi^{2} / 2}, \\
\phi_{B^{\prime}}=f_{n} d_{n} c_{n} H_{n}(\xi) e^{-\xi^{2} / 2},
\end{gathered}
$$

where $H_{n}(\xi)$ is the Hermite polynomial of order $n$ and

$$
\begin{gathered}
c_{n}=1 /\left(n ! 2^{n} \sqrt{\pi}\right)^{1 / 2}, \\
f_{n}=\frac{\left[\left(\alpha_{n}-\delta\right)^{2}-2 n\right]}{t^{\prime}\left(\alpha_{n}-\delta\right)}, \\
d_{n}=\left\{f_{n}^{2}\left[1+\frac{2(n+1)}{\left(\alpha_{n}+\delta\right)^{2}}\right]+1+\frac{2 n}{\left(\alpha_{n}-\delta\right)^{2}}\right\}^{-1 / 2} .
\end{gathered}
$$

Notice that the eigenstates are determined by the quantum numbers $\left(k_{y}, n\right)$, while the spectrum is independent of $k_{y}$.

\section{B. Symmetric gauge}

In problems with cylindrical symmetry, it is advantageous to use the symmetric gauge $\mathbf{A}=(0, B \rho / 2,0)$. In this case, the corresponding solutions for the four-component spinors $\Psi$ are written as ${ }^{16,22}$

$$
\Psi(\rho, \theta)=\left(\begin{array}{c}
\phi_{A}(\rho) e^{i m \theta} \\
i \phi_{B}(\rho) e^{i(m-1) \theta} \\
\phi_{B^{\prime}}(\rho) e^{i m \theta} \\
i \phi_{A^{\prime}}(\rho) e^{i(m+1) \theta}
\end{array}\right),
$$

where $m$ is the angular momentum label. The radial dependence of the spinor components is described, in dimensionless units, by

$$
\frac{1}{\sqrt{2}}\left[\frac{d}{d \xi}+\frac{m}{\xi}+\xi\right] \phi_{A}=(\alpha-\delta) \phi_{B},
$$

$$
\begin{gathered}
\frac{1}{\sqrt{2}}\left[\frac{d}{d \xi}-\frac{(m-1)}{\xi}-\xi\right] \phi_{B}=-(\alpha-\delta) \phi_{A}+t^{\prime} \phi_{B^{\prime}}, \\
\frac{1}{\sqrt{2}}\left[\frac{d}{d \xi}+\frac{(m+1)}{\xi}+\xi\right] \phi_{A^{\prime}}=-(\alpha+\delta) \phi_{B^{\prime}}+t^{\prime} \phi_{A}, \\
\frac{1}{\sqrt{2}}\left[\frac{d}{d \xi}-\frac{m}{\xi}-\xi\right] \phi_{B^{\prime}}=(\alpha+\delta) \phi_{A^{\prime}},
\end{gathered}
$$

where now $\xi=\sqrt{2} \rho / 2 \ell_{B}$, and the other terms are defined as before.

Following the same procedure as in the previous case, one can obtain an equation that is identical to that of a harmonic oscillator

$$
\left[-\frac{1}{2}\left(\frac{d^{2}}{d \xi^{2}}+\frac{1}{\xi} \frac{d}{d \xi}-\frac{m^{2}}{\xi^{2}}\right)+m+\frac{\xi^{2}}{2}\right] \phi_{A}=2 \gamma_{ \pm}(\alpha) \phi_{A},
$$

where

$$
\gamma_{ \pm}(\alpha)=\left(n^{\prime}+1 / 2\right),
$$

with $n^{\prime}=n+(|m|+m) / 2, n=0,1,2, \ldots$, and $\gamma_{ \pm}(\alpha)$ is given by Eq. (12). The LL spectrum is determined by

$$
\left[(\alpha+\delta)^{2}-2\left(n^{\prime}+1\right)\right]\left[(\alpha-\delta)^{2}-2 n^{\prime}\right]-\left(\alpha^{2}-\delta^{2}\right) t^{\prime 2}=0,
$$

which is identical to Eq. (13) except that here the Landau index $n^{\prime}$ is used instead of $n$.

It is evident, from Eq. (18), that the spinor components $\phi_{A}$ and $\phi_{B^{\prime}}$ can be obtained in terms of generalized Laguerre polynomials. For $m>0$, we obtain

$$
\begin{gathered}
\phi_{A}=C_{A n}^{m} \xi^{|m|} e^{-\xi^{2} / 2} L_{n}^{|m|}\left(\xi^{2}\right), \\
\phi_{B}=\frac{\sqrt{2 n^{\prime}}}{\alpha_{n^{\prime}}-\delta} C_{A n}^{m-1} \xi^{|m|-1} e^{-\xi^{2} / 2} L_{n}^{|m|-1}\left(\xi^{2}\right), \\
\phi_{A^{\prime}}=-\frac{\sqrt{2\left(n^{\prime}+1\right)}}{\alpha_{n^{\prime}}+\delta} C_{B^{\prime} n}^{m+1} \xi^{|m|+1} e^{-\xi^{2} / 2} L_{n}^{|m|+1}\left(\xi^{2}\right),
\end{gathered}
$$




$$
\phi_{B^{\prime}}=C_{B^{\prime} n}^{m} \xi^{|m|} e^{-\xi^{2} / 2} L_{n}^{|m|}\left(\xi^{2}\right) .
$$

For $m<0$, the result is

$$
\begin{gathered}
\phi_{A}=C_{A n}^{m} \xi^{|m|} e^{-\xi^{2} / 2} L_{n}^{|m|}\left(\xi^{2}\right), \\
\phi_{B}=-\frac{\sqrt{2 n^{\prime}}}{\alpha_{n^{\prime}}-\delta} C_{A n-1}^{m+1} \xi^{|m|+1} e^{-\xi^{2} / 2} L_{n-1}^{|m|+1}\left(\xi^{2}\right), \\
\phi_{A^{\prime}}=\frac{\sqrt{2\left(n^{\prime}+1\right)}}{\alpha_{n^{\prime}}+\delta} C_{B^{\prime} n+1}^{m+1} \xi^{|m|-1} e^{-\xi^{2} / 2} L_{n+1}^{|m|-1}\left(\xi^{2}\right), \\
\phi_{B^{\prime}}=C_{B^{\prime} n}^{m} \xi^{|m|} e^{-\xi^{2} / 2} L_{n}^{|m|}\left(\xi^{2}\right),
\end{gathered}
$$

where

$$
C_{A n}^{m}=d_{n} /[n ! /(n+|m|) !]^{1 / 2}, \quad C_{B^{\prime} n}^{m}=f_{n} C_{A n}^{m} ;
$$

the other constants are as before.

\section{Special cases}

(i) $t^{\prime}=\delta=0$. This is the case of a single graphene layer or two uncoupled, unbiased layers. The solutions are then

$$
\alpha_{n^{\prime}}= \pm \sqrt{2\left(n^{\prime}+1\right)}, \quad \alpha_{n^{\prime}}= \pm \sqrt{2 n^{\prime}},
$$

which are the well known expressions for the LL in a single layer of graphene.

(ii) $\delta=0$. This is the case of an unbiased bilayer. The explicit solutions are

$$
\begin{aligned}
\alpha_{n^{\prime}}= & \pm \frac{t^{\prime}}{\sqrt{2}}\left[1+\frac{2}{t^{\prime 2}}\left(2 n^{\prime}+1\right)\right. \\
& \pm \sqrt{\left.\left[1+\frac{2}{t^{\prime 2}}\left(2 n^{\prime}+1\right)\right]^{2}-\frac{16}{t^{\prime 4}} n^{\prime}\left(n^{\prime}+1\right)\right]^{1 / 2}} .
\end{aligned}
$$

This expression can be simplified by expanding the internal square root. By taking the negative sign, one obtains

$$
\alpha_{n^{\prime}}= \pm \frac{2}{t^{\prime}} \sqrt{n^{\prime}\left(n^{\prime}+1\right)} /\left[1+\frac{2}{t^{\prime 2}}\left(2 n^{\prime}+1\right)\right]^{1 / 2},
$$

which is valid for $n^{\prime} / t^{\prime 2} \ll 1$. By assuming $1 /\left[1+\left(2 / t^{\prime 2}\right)\right.$ $\left.\times\left(2 n^{\prime}+1\right)\right]^{1 / 2} \approx 1$, Eq. (26) is simplified to

$$
\alpha_{n^{\prime}}= \pm \frac{2}{t^{\prime}} \sqrt{n^{\prime}\left(n^{\prime}+1\right)}
$$

which is the expression previously obtained in the context of the two-band continuum model. ${ }^{19}$ By taking the positive sign in front of the internal square root in Eq. (25), one finds the higher-energy LL that arises from the upper band at $E=t$. For $n^{\prime}=0$, that gives

$$
\alpha_{0}= \pm \sqrt{t^{\prime 2}+2} \text {. }
$$

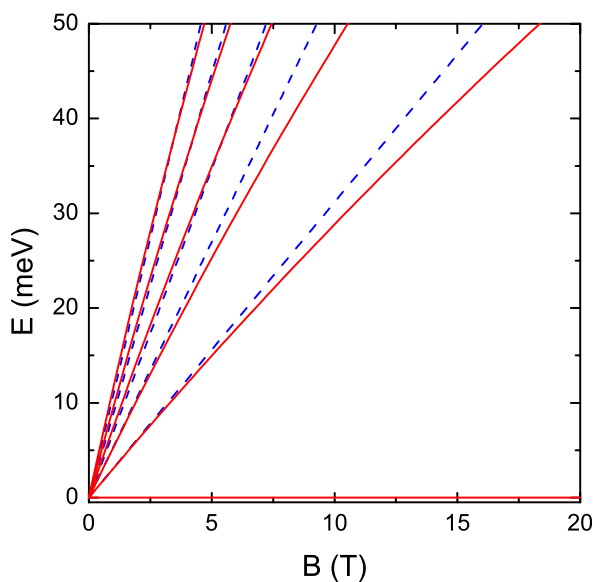

FIG. 2. (Color online) Landau levels $(n=0-5)$ in an unbiased graphene bilayer as a function of the magnetic field. The solid, red lines result from a numerical solution of Eq. (25) and the dashed, blue ones are the approximate results from Eq. (27).

\section{NUMERICAL RESULTS FOR THE ENERGY SPECTRUM}

Figure 2 shows the first six positive energy LL as a function of the magnetic field $B$ for an unbiased graphene bilayer. The figure shows that as $B$ increases, Eq. (27) consistently overestimates the values of the energy levels (dashed lines) as compared to the exact results obtained from Eq. (25) (solid) [which coincide with those from the approximate expression Eq. (26)]. The discrepancy increases with $B$ and, for the parameters usually observed experimentally ( $t$ $=0.4 \mathrm{eV}, v_{F} \approx 1.0 \times 10^{6} \mathrm{~m} / \mathrm{s}$ ), can attain $5 \mathrm{meV}$ for $B=15 \mathrm{~T}$ and $n=1$. This result was confirmed by a numerical calculation based on a discretization of Eq. (17). Recent experimental studies have measured the LL spectrum in bilayers of graphene for fields up to $12 \mathrm{~T} .{ }^{23}$ At these fields, the discrepancy between the four-band and two-band results are $\approx 10 \%$ for the $n=1 \mathrm{LL}$. This is below the energy resolution of the current experimental techniques. However, for stronger fields, the difference should become large enough to become detectable. In addition, the presence of the LL associated with the higher-energy band at $E \approx 0.4 \mathrm{eV}$ may be detectable by the scanning tunneling technique discussed in Ref. 23

Equation (20) shows that the charge-conjugation symmetry between electrons and holes is preserved for the Landau states in a single layer $(t=0, \delta=0)$ as well as in the unbiased double layer $(\delta=0)$, since the equation is unchanged under the transformation $\alpha \rightarrow-\alpha$. On the other hand, that ceases to be the case for a biased bilayer of graphene. Figure 3 shows the first six low-energy LL for a biased bilayer with $U_{1}=$ $-U_{2}=50 \mathrm{meV}$. The positive (negative) energy levels are shown in Fig. 3(a) [Fig. 3(b)], and the results show a nonmonotonic dependence on the magnetic field. To each index $n$ there correspond four solutions, two negative and two positive, with the low-energy branches being equal to $|\delta|$ for $B$ $=0$, whereas the high-energy solutions arise from the bands at $|\alpha|=t^{\prime}$ (not shown). The only exception is the $n=0$ state, for which, in the case of the $K$ valley, there is no positive solution at the vicinity of $\delta$ (the opposite is true at the vicin- 

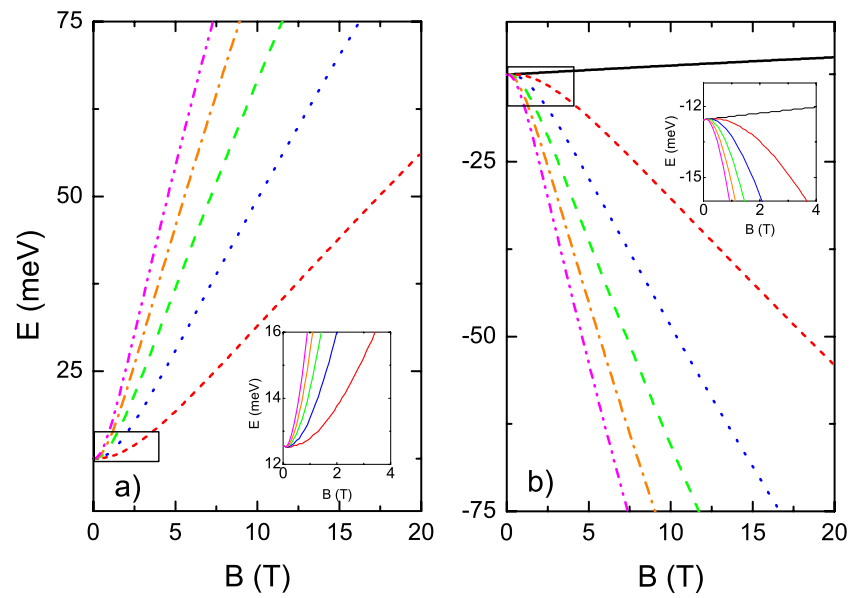

FIG. 3. (Color online) (a) Positive-energy Landau levels in a biased graphene bilayer as a function of the magnetic field $B$. (b) As in (a) for negative-energy levels. In both cases, $U_{1}=-U_{2}=50 \mathrm{meV}$ and $n=0$ (solid line), $n=1$ (dashed lines), $n=2$ (dotted), $n=3$ (long dashed), $n=4$ (dash dotted), and $n=5$ (dot-dot-dashed lines). The rectangular areas are enlarged in the insets.

ity of the $K^{\prime}$ valley). The figure shows the LL corresponding to $n=0$ (black solid line), $n=1$ (red dashed lines), $n=2$ (blue dotted line), $n=3$ (green long-dashed line), $n=4$ (orange dotdashed line), and $n=5$ (magenta dot-dot-dashed lines). One striking feature in the spectrum is the appearance of crossings and energy minima in some magnetic field regions shown in the insets. These features arise as the potential difference (i.e., the gap parameter) between the layers is increased, with the energy minima and the crossings initially appearing at very small values of $B$. As $\Delta U$ increases, the minima in each branch occur at larger magnetic fields and lower energies. These results can be explained by taking into account the band structure of the biased graphene bilayer. The introduction of a magnetic field in a $2 \mathrm{D}$ system constrains the electronic states to closed cyclotron orbits, which, in turn, causes an energy quantization in which the energy eigenvalues correspond to momentum states that are integer multiples of $h / \ell_{B}$. For a system with a parabolic band, this gives rise to a linear dependence of the LL on the magnetic field. For a single graphene layer, on the other hand, the linear momentum dependence of the energy introduces a $\sqrt{B}$ dependence of the LL spectrum. In a biased graphene bilayer, the electron dispersion has a more complicated, "mexican hat" shape (see Fig. 1) that becomes more pronounced as $\Delta U$ becomes larger. For $B=0$, this dispersion then allows the existence of pairs of degenerate states with different values of momenta, which, for finite $B$, can become degenerate LL with different Landau indices. By setting $n^{\prime}=0$ in Eq. (20), we obtain, for $\alpha$ values close to $-\delta$,

$$
\alpha_{0}=-\delta \frac{\left(1-2 \beta / t^{\prime 2}\right)}{\left(1+2 \beta / t^{\prime 2}\right)}
$$

The introduction of bias, together with the presence of a magnetic field, breaks the inversion symmetry of the bilayer, which, in turn, breaks the charge-conjugation symmetry. A

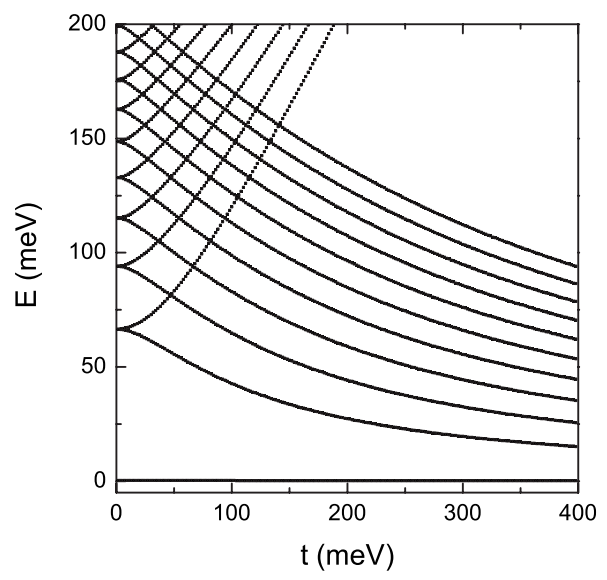

FIG. 4. Landau levels in an unbiased graphene bilayer, for $B$ $=5 \mathrm{~T}$, as a function of the interlayer coupling parameter $t$.

similar effect is obtained for the case of states in a positiondependent confining potential. ${ }^{16}$ This electron-hole asymmetry is particularly evident in the lowest LLs, which are not distributed symmetrically around $E=U_{0}$, i.e., $E_{n} \neq-E_{-n}$, for $U_{0}=0$, except for $\Delta U \approx 0$.

Previous studies of LLs in bilayer graphene that were based on a continuum model have used a two-band approximation, which is expected to be accurate for large coupling interlayer strengths $t$. The four-band model used here allows one to investigate the whole range of interlayer interaction strengths. Figure 4 shows the first ten LLs for an unbiased graphene bilayer as a function of the coupling parameter $t$. The $t=0$ limit corresponds to the case of two uncoupled single layers, and the spectrum is given by Eq. (24). As the coupling increases, the twofold degeneracy of the levels is lifted, with the higher-energy levels being shifted toward $E$ $=t$, and several crossings occur. The figure also shows that the energy difference between the lowest LLs decreases for increasing $t$, with the $n=0$ LL remaining unaffected by the coupling in the absence of bias.

The dependence of the spectrum on the energy gap can be seen in Fig. 5, which shows the LL corresponding to $n=0$ (black solid line), $n=1$ (red dashed lines), $n=2$ (blue dotted), $n=3$ (green long dashed), $n=4$ (orange dash dotted), and $n$ $=5$ (magenta dot-dot-dashed lines). One striking feature of this result is the reduction of the energy difference between some of the LL as the gap increases, with the eventual appearance of degeneracies. That can be explained by considering the fact that as $\Delta U$ increases, the band structure of the bilayer evolves from an approximately parabolic dispersion to the approximately fourth-order polynomial dependence on the momentum. From Eq. (20), one can easily show that degeneracies will occur between LL with indices $n_{1}$ and $n_{2}$ for energies and fields that satisfy the relation

$$
n_{1}+n_{2}=\alpha_{n_{1}}^{2}+\delta^{2}-1 \text {. }
$$

\section{OSCILLATOR STRENGTH}

The oscillator strength $\left|\left\langle\Psi^{\dagger}\left|\rho e^{i \phi}\right| \Psi\right\rangle\right|^{2}$ for electric dipole transitions between the LLs is given by 


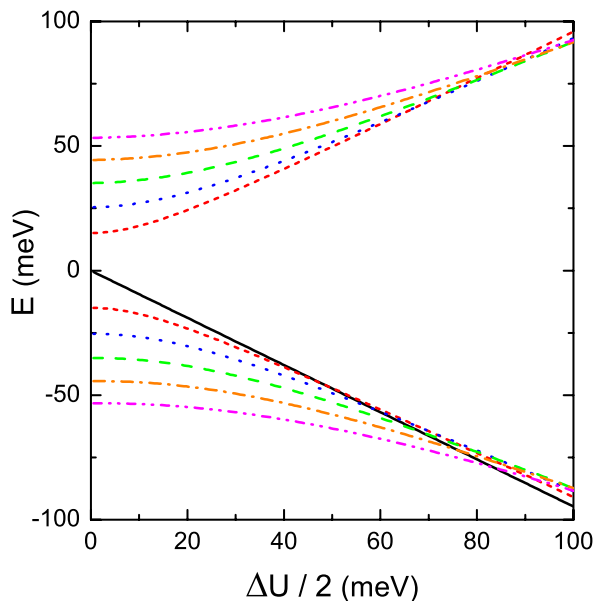

FIG. 5. (Color online) Landau levels in a graphene bilayer as a function of the difference $\Delta U$ in potential between the layers for $B=5 \mathrm{~T}: n=0$ (black solid line), $n=1$ (red dashed lines), $n=2$ (blue dotted lines), $n=3$ (green long-dashed lines), $n=4$ (orange dashdotted lines), and $n=5$ (magenta dot-dot-dashed lines).

$$
T=\left(T_{A}+T_{B}+T_{A^{\prime}}+T_{B^{\prime}}\right)^{2},
$$

where

$$
T_{C}=\int \phi_{C, l} \xi \phi_{C, l^{\prime}} d \xi
$$

with $C=A, B, A^{\prime}$, and $B^{\prime}$. These matrix elements were calculated in the case of the Landau gauge as

$$
\begin{gathered}
T_{A}=d_{l} d_{l^{\prime}}\left(\sqrt{l} \delta_{l, l^{\prime}+1}+\sqrt{l+1} \delta_{l, l^{\prime}-1}\right) / \sqrt{2} \\
T_{B}=\frac{d_{l} d_{l^{\prime}} 2 \sqrt{l l^{\prime}}}{\left(\alpha_{l}-\delta\right)\left(\alpha_{l^{\prime}}-\delta\right)} \frac{\left(\sqrt{l-1} \delta_{l, l^{\prime}+1}+\sqrt{l} \delta_{l, l^{\prime}-1}\right)}{\sqrt{2}} \\
T_{A^{\prime}}=d_{l} d_{l^{\prime}} f_{l} f_{l^{\prime}} \frac{2 \sqrt{\left(l^{\prime}+1\right)(l+1)}}{\left(\alpha_{l}+\delta\right)\left(\alpha_{l^{\prime}}+\delta\right)} \frac{\left(\sqrt{l+1} \delta_{l, l^{\prime}+1}+\sqrt{l+2} \delta_{l, l^{\prime}-1}\right)}{\sqrt{2}}, \\
T_{B^{\prime}}=d_{l} d_{l^{\prime}} f_{l} f_{l^{\prime}}\left(\sqrt{l} \delta_{l, l^{\prime}+1}+\sqrt{l+1} \delta_{l, l^{\prime}-1}\right) / \sqrt{2}
\end{gathered}
$$

where $l$ and $l^{\prime}$ are Landau indices of the different eigenstates. The selection rule is $\delta l=|l|-\left|l^{\prime}\right|= \pm 1$. Here, $\left(l, l^{\prime}\right)$ indicates a transition from a LL with index $l$ to another with index $l^{\prime}$; the negative sign is used to represent a transition from a holelike LL. The oscillator strengths for some dipole-allowed transitions between LL are shown in Fig. 6, for a biased bilayer (a) with $U_{1}=-U_{2}=50 \mathrm{meV}$ and $B=10 \mathrm{~T}$ as well as for an uniased bilayer (b). The effect of the bias is most clear in the shift in energy of the $(0,1)$ and $(-1,-2)$ transitions, caused by the gap in the spectrum. On the other hand, one can also observe a decrease in the energies associated with transitions between the higher LL.

Figure 7(a) shows the energy $\Delta E$ for some transitions between LLs in a biased graphene bilayer as a function of the magnetic field, for $U_{1}=-U_{2}=50 \mathrm{meV}$, namely, $(0,1)$ (black
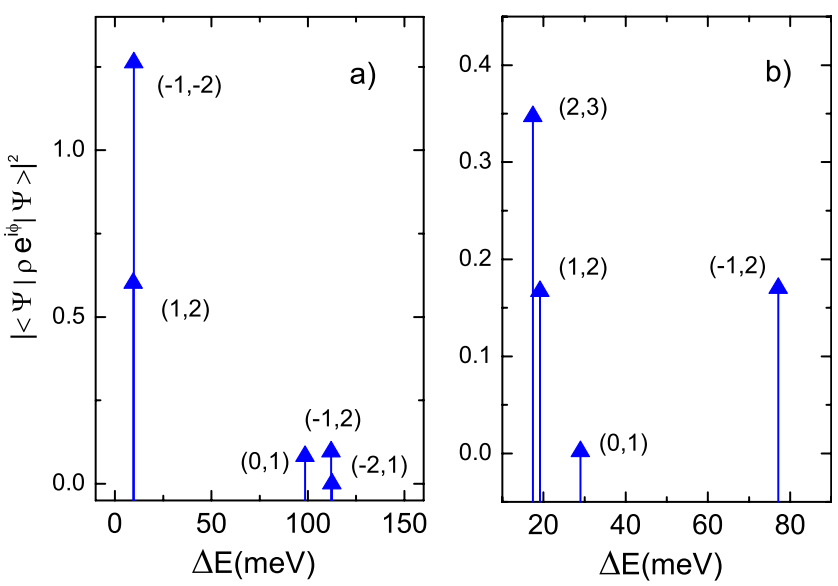

FIG. 6. (Color online) (a) Oscillator strengths for electric dipole transitions in a biased bilayer of graphene $(\Delta U=100 \mathrm{meV})$ for $B$ $=10 \mathrm{~T}$. (b) As in (a) for an unbiased $(\Delta U=0 \mathrm{meV})$ bilayer.

dashed lines), (1, 2) (blue dotted line), $(-1,-2)$ (black solid), and $(-1,2)$ (red solid line). In contrast to the case of the unbiased bilayer as well as the single layer, $\Delta E$ is seen to be weakly dependent on the field for $B<5 \mathrm{~T}$. For the $(1,2)$ and $(-1,-2)$ transitions, the energy reaches negative values due to the existence of LL crossings (see Fig. 3). In addition, a difference in the energy of these transitions is found (see inset). The results are obtained by subtracting the energies associated with the initial and final LLs. However, recent theoretical work has indicated that many-body corrections may cause a substantial modification of the transition energies. ${ }^{24}$ Figure 7(b) shows the oscillator strengths for the same transitions as in the left panel, as a function of the magnetic field. These results show that, for small fields, e.g., for $B<10 \mathrm{~T}$, the oscillator strength associated with the $(-1,2)$ transition is slightly larger than that of the $(0,1)$ transition, whereas for larger fields, the latter becomes stron-
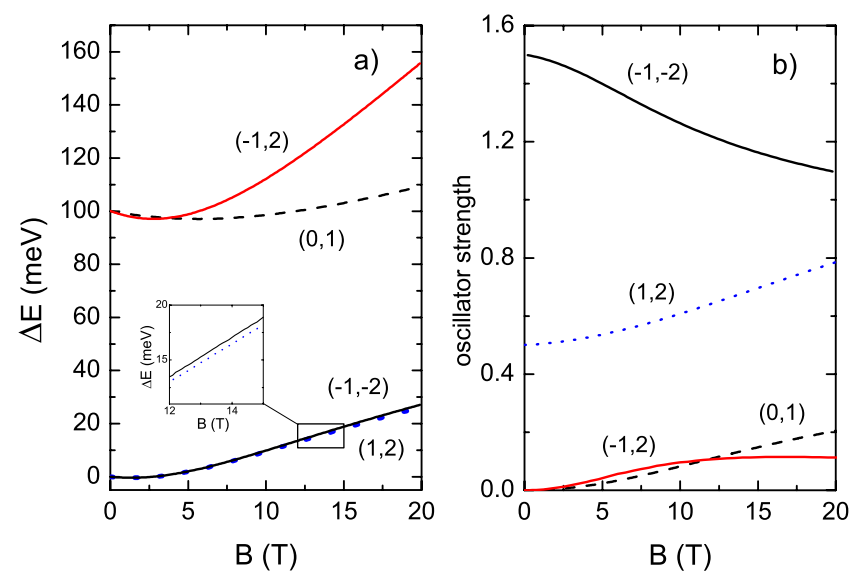

FIG. 7. (Color online) (a) Transition energies in a biased $(\Delta U$ $=100 \mathrm{meV}$ ) graphene bilayer, as a function of the magnetic field $B$, for the dipole-allowed transitions: $(0,1)$ (black dashed line), $(1,2)$ (blue dotted line), $(-1,-2)$ (black solid line), and $(-1,2)$ (red solid line). (b) Oscillator strengths vs field $B$ for the transitions described in (a). 


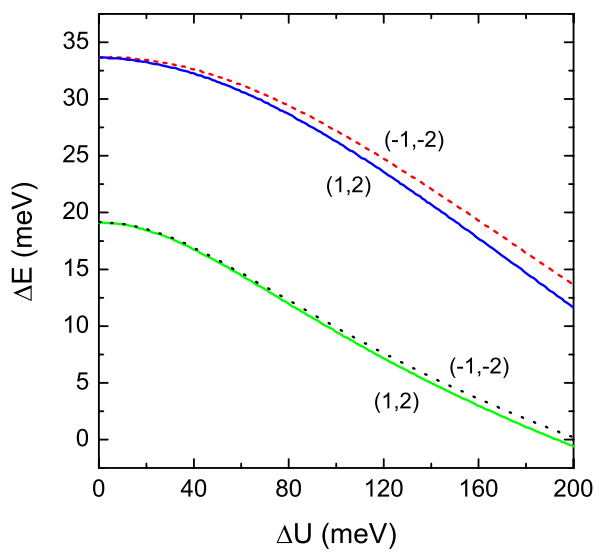

FIG. 8. (Color online) Transition energies as a function of $\Delta U$ for the $(1,2)$ (blue solid line) and $(-1,-2)$ (red dashed line) transitions for $B=20 \mathrm{~T}$, and $(1,2)$ (green solid line) and $(-1,-2)$ (black dotted line) for $B=10 \mathrm{~T}$.

ger. The curve for the $(-1,2)$ transition intercepts the result for the $(0,1)$ one at $B \approx 11.6 \mathrm{~T}$, which corresponds to $E_{1}=$ $-U_{1}$ [see Fig. 3(b)]. As in Fig. 7(a), the results show a significant asymmetry between the electron $(1,2)$ and hole $(-1,-2)$ intraband transitions.

This difference between the electron and hole intraband transitions becomes larger as the gap parameter $(\Delta U)$ is increased, as shown in Fig. 8, which displays the energies for the $(1,2)$ (solid blue line) and $(-1,-2)$ (dashed red line) transitions as a function of $\Delta U$ for $B=20 \mathrm{~T}$, and $(1,2)$ (solid green line) and $(-1,-2)$ (dotted black line) for $B=10 \mathrm{~T}$. At $\Delta U=200 \mathrm{meV}$, the energy difference reaches $\approx 3 \mathrm{meV}$ for $B=20 \mathrm{~T}$, and $\approx 0.8 \mathrm{meV}$ for $B=10 \mathrm{~T}$. For the $B=10 \mathrm{~T}$ case, the energy difference reaches zero and eventually becomes negative, due to the crossing of the LL. The asymmetry between the electron and hole intraband transitions is also particularly evident in their oscillator strength values, as shown in Fig. 9, which presents the oscillator strengths for the transitions discussed in Fig. 7, as a function of the gap parameter, for $B=5 \mathrm{~T}$. The results show that the presence of a gap causes a significant reduction of the oscillator strength for the $(0,1)$ and $(1,2)$ transitions, whereas the result for $(-1,2)$ shows a slight increase at small fields, reaching a maximum at $\Delta U \approx 25 \mathrm{meV}$ with a subsequent decrease. On the other hand, the curve for the $(-1,-2)$ transition has a striking increase in comparison with the result for $\Delta U=0$.

Figure 10(a) shows the energies for the $(1,2)$ and $(-1$, -2) transitions (i.e., the cyclotron resonance transitions) as a function of field, for several values of the gap parameter: $\Delta U=0$ (black solid line), $\Delta U=25 \mathrm{meV}$ (red dashed lines), $50 \mathrm{meV}$ (blue dotted lines), $100 \mathrm{meV}$ (green dot-dashed lines), and $\Delta U=200 \mathrm{meV}$ (magenta dot-dot-dashed lines). For $\Delta U=0$, the $\Delta E$ for the two transitions coincides, whereas in the other cases, the energy associated with the hole intraband transition is always higher than the corresponding electron transition. Note that the $\Delta U=0$ result only exhibits linear $B$ dependence, as predicted by the simple expression Eq. (27), for small $B$ fields. With increasing $\Delta U$, the cyclotron energy decreases, and for small $B$ values, $\Delta E$ becomes even

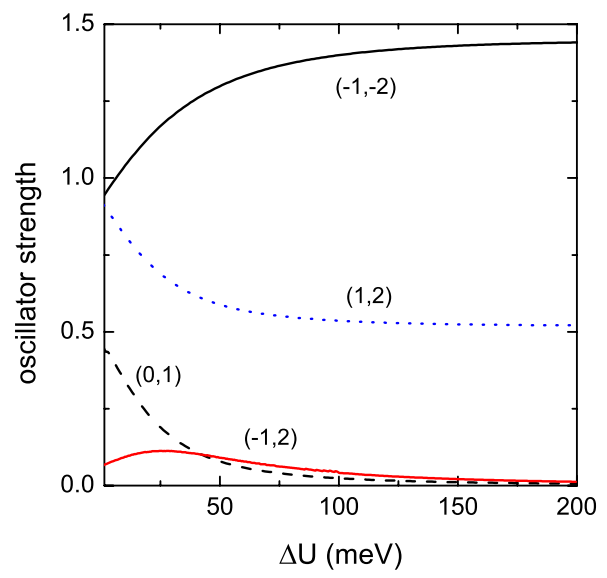

FIG. 9. (Color online) Oscillator strengths for dipole-allowed transitions in a graphene bilayer as a function of the interlayer potential difference $\Delta U$ for the $(0,1)$ (black dashed line), $(1,2)$ (blue dotted line), $(-1,-2)$ (black solid), and $(-1,2)$ (red solid line) transitions at $B=5 \mathrm{~T}$.

negative, indicating a reversion of the order of the LL. These qualitative changes in $\Delta E$ should be observable in a cyclotron resonance experiment.

The oscillator strengths for the transitions shown in the left panel are displayed in Fig. 10(b). It is seen that for the gapless system, the oscillator strengths are independent of the magnetic field. On the other hand, as the gap increases, the results show a strong asymmetry that increases as $\Delta U$ becomes larger. In all cases, as $B$ increases, the results approach the oscillator strengths for the $\Delta U=0$ case. A similar behavior is also found for the other $(l, l+1)$ and $(-l,-l-1)$ transitions, albeit with smaller discrepancy in the energies.

The asymmetry between the electron and hole intraband transitions can be explained by taking into account the func-
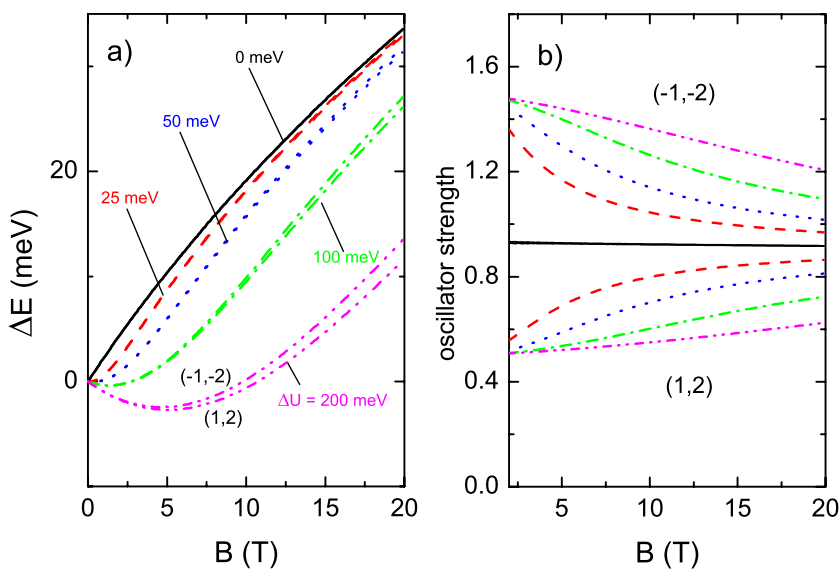

FIG. 10. (Color online) (a) Energies of the $(1,2)$ and $(-1,-2)$ (upper curves of each doublet) transitions as a function of magnetic field for $\Delta U=0$ (black solid line), $\Delta U=25 \mathrm{meV}$ (red dashed lines), $\Delta U=50 \mathrm{meV}$ (blue dotted lines), $\Delta U=100 \mathrm{meV}$ (green dot-dashed lines), and $\Delta U=200 \mathrm{meV}$ (magenta dot-dot-dashed lines). (b) The oscillator strengths for the transitions shown in (a) as a function of magnetic field. The results below 0.92 correspond to the $(1,2)$ transitions. 
tional dependence of the amplitude factors of the spinor components on the energy. These terms, in the presence of a gap, introduce an asymmetry between the envelope functions of electrons and holes, thus modifying the transition probabilities. This effect is, in turn, a consequence of the breaking of the inversion symmetry of the biased bilayer.

\section{SUMMARY AND CONCLUSIONS}

Using a four-band model, we obtained analytical expressions for the Landau levels and the eigenfunctions in a biased graphene bilayer in the presence of an external perpendicular magnetic field $B$. In doing so, we also extended previous results for the discrete spectrum of an unbiased graphene layer based on a two-band model. ${ }^{19}$ Further, we obtained the selection rules for electric dipole transitions and evaluated the associated oscillator strengths which in Ref. 20 were evaluated only for the unbiased grapheme.

Relative to an unbiased bilayer, the introduction of a bias modifies the spectrum considerably by reducing the energy for transitions between two electron states while at the same time increasing the energy for transitions between an electron and a hole state (see Figs. 5 and 6). Also noteworthy is the modification of the electronic band structure of the bilayer caused by the bias, which leads to the breaking of electron-hole symmetry, the appearance of degeneracies in the LL spectrum, and qualitatively different dependency of the transition energy $\Delta E$ on the magnetic field, which, in turn, can strongly modify the dipole transition probabilities. These effects can be probed by cyclotron resonance measurements, ${ }^{7}$ scanning tunneling spectroscopy ${ }^{23}$ and farinfrared spectroscopy. ${ }^{6}$ Future work may take into account the effects of spin as well as electron-electron interactions, which may become particularly important at high magnetic fields. ${ }^{21,25}$

\section{ACKNOWLEDGMENTS}

This work was supported by the Brazilian Council for Research (CNPq), BOF-UA, the Flemish Science Foundation (FWO-Vl), the Belgian Science Policy (IAP), and the Canadian NSERC Grant No. OGP0121756.
${ }^{1}$ Y. Zheng and T. Ando, Phys. Rev. B 65, 245420 (2002).

${ }^{2}$ K. S. Novoselov, A. K. Geim, S. V. Morozov, D. Jiang, Y. Zhang, S. V. Dubonos, I. V. Grigorieva, and A. A. Firsov, Science 306, 666 (2004).

${ }^{3}$ K. S. Novoselov, A. K. Geim, S. V. Morozov, D. Jiang, M. I. Katsnelson, I. V. Grigorieva, S. V. Dubonos, and A. A. Firsov, Nature (London) 438, 197 (2005).

${ }^{4}$ Y. Zhang, Y. W. Tan, H. L. Stormer, and P. Kim, Nature (London) 438, 201 (2005).

${ }^{5}$ V. P. Gusynin and S. G. Sharapov, Phys. Rev. Lett. 95, 146801 (2005).

${ }^{6}$ M. L. Sadowski, G. Martinez, M. Potemski, C. Berger, and W. A. de Heer, Phys. Rev. Lett. 97, 266405 (2006).

${ }^{7}$ R. S. Deacon, K. C. Chuang, R. J. Nicholas, K. S. Novoselov, and A. K. Geim, arXiv:0704.0410v2 (unpublished).

${ }^{8}$ B. Partoens and F. M. Peeters, Phys. Rev. B 74, 075404 (2006).

${ }^{9}$ T. Ohta, A. Bostwick, J. L. McChesney, T. Seyller, K. Horn, E. Rotenberg, arXiv:cond-mat/0612173v1 (unpublished).

${ }^{10}$ M. I. Katsnelson, K. S. Novoselov, and A. K. Geim, Nat. Phys. 2, 620 (2006).

${ }^{11}$ J. M. Pereira, Jr., V. Mlinar, F. M. Peeters, and P. Vasilopoulos, Phys. Rev. B 74, 045424 (2006).

${ }^{12}$ V. V. Cheianov and V. I. Fal'ko, Phys. Rev. B 74, 041403(R) (2006).
${ }^{13}$ J. Milton Pereira, Jr., P. Vasilopoulos, and F. M. Peeters, Appl. Phys. Lett. 90, 132122 (2007).

${ }^{14}$ T. Ohta, A. Bostwick, T. Seyller, K. Horn, and E. Rotenberg, Science 313, 951 (2006).

${ }^{15}$ H. Min, B. Sahu, S. K. Banerjee, and A. H. MacDonald, Phys. Rev. B 75, 155115 (2007).

${ }^{16}$ J. Milton Pereira, Jr., P. Vasilopoulos, and F. M. Peeters, Nano Lett. 7, 946 (2007).

${ }^{17}$ E. McCann, Phys. Rev. B 74, 161403(R) (2006).

${ }^{18}$ E. V. Castro, K. S. Novoselov, S. V. Morozov, N. M. R. Peres, J. M. B. Lopes dos Santos, J. Nilsson, F. Guinea, A. K. Geim, and A. H. Castro Neto, arXiv:cond-mat/0611342 (unpublished).

${ }^{19}$ E. McCann and V. I. Fal'ko, Phys. Rev. Lett. 96, 086805 (2006).

${ }^{20}$ D. L. Abergel and V. I. Fal'ko, Phys. Rev. B 75, 155430 (2007).

${ }^{21}$ T. Stauber, N. M. R. Peres, F. Guinea, and A. H. Castro Neto, Phys. Rev. B 75, 115425 (2007).

${ }^{22}$ D. P. DiVincenzo and E. J. Mele, Phys. Rev. B 29, 1685 (1984).

${ }^{23}$ G. Li and E. Y. Andrei, arXiv:0705.1185, Nat. Phys. (to be published).

${ }^{24}$ A. Iyengar, J. Wang, H. A. Fertig, and L. Brey, Phys. Rev. B 75, 125430 (2007).

${ }^{25}$ Z. Jiang, E. A. Henriksen, L. C. Tung, Y.-J. Wang, M. E. Schwartz, M. Y. Han, P. Kim, and H. L. Stormer, Phys. Rev. Lett. 98, 197403 (2007). 